Article

\title{
Hydroxyl Ion Diffusion through Radicular Dentine When Calcium Hydroxide Is Used under Different Conditions
}

\author{
Michael Cai, Paul Abbott * (D) and Jacqueline Castro Salgado \\ UWA Dental School, the University of Western Australia, Crawley, WA 6009, Australia; \\ michaelhcai@gmail.com (M.C.); jacky.castro-salgado@uwa.edu.au (J.C.S.) \\ * Correspondence: paul.v.abbott@uwa.edu.au; Tel.: +61-8-6457-7665 \\ Received: 22 November 2017; Accepted: 15 January 2018; Published: 17 January 2018
}

\begin{abstract}
Calcium hydroxide's anti-bacterial action relies on high $\mathrm{pH}$. The aim here was to investigate hydroxyl ion diffusion through dentine under different conditions. Teeth were divided into control $(n=4)$ and four experimental groups $(n=10)$ : Group 1-no medicament; Group 2-Calmix; Group 3-Calmix/Ledermix; Group 4-Calasept Plus/Ledermix; Group 5-Pulpdent/smear layer. Deep (inner dentine) and shallow (outer dentine) cavities were cut into each root. $\mathrm{pH}$ was measured in these cavities for 12 weeks. The inner and outer dentine $\mathrm{pH}$ in Group 2 was significantly higher than all groups. Inner dentine $\mathrm{pH}$ in Group 3 was slightly higher than that in Group 4 initially but subsequently comparable. After Day 2, Group 5 had significantly lower $\mathrm{pH}$ than Groups 3 and 4. The outer dentine $\mathrm{pH}$ in Group 3 started higher than that in Groups 4 and 5, but by Day 28 the difference was insignificant. The time for the inner dentine to reach maximum $\mathrm{pH}$ was one week for Group 2 and four weeks for Groups 3 and 4. The time for the outer dentine to reach maximum $\mathrm{pH}$ was eight weeks for all experimental groups. Mixing different $\mathrm{Ca}(\mathrm{OH})_{2}$ formulations with Ledermix gave similar hydroxyl ion release but $\mathrm{pH}$ and total diffusion was lower than $\mathrm{Ca}(\mathrm{OH})_{2}$ alone. The smear layer inhibited diffusion.
\end{abstract}

Keywords: calcium hydroxide; diffusion; Ledermix paste; endodontics; root canal treatment

\section{Introduction}

The main aim of root canal treatment of infected teeth is to disinfect the root canal system. However, this cannot be reliably achieved by root canal instrumentation with files and irrigation with anti-bacterial solutions, so the supporting action of intracanal medicaments is required [1-3]. Byström and Sundqvist reported that they were able to cultivate bacteria from the root canals of $28 \%$ of the tested teeth, even after three visits of root canal instrumentation and irrigation with sodium hypochlorite $(\mathrm{NaOCl})$, ethylenediaminetetraacetic acid (EDTA), or a combination of these irrigants [1]. They also reported an increase in bacterial numbers between appointments when no anti-bacterial medicament was placed. Therefore, the use of inter-appointment antibacterial medicaments in the root canal system is necessary to predictably achieve bacteria-free root canals during endodontic treatment [1-3].

Calcium hydroxide $\left(\mathrm{Ca}(\mathrm{OH})_{2}\right)$ is one of the most popular root canal medicaments and it comes in different forms and combinations [4]. $\mathrm{Ca}(\mathrm{OH})_{2}$ has several roles during root canal treatment, namely, (A) antibacterial action [5], (B) the ability to stimulate hard tissue formation (e.g., apexification [6] and apexogenesis [7]), (C) the ability to dissolve tissue [8], and (D) the ability to cause intratubular mineralization in dentine [9]. The anti-bacterial action is a direct effect of the hydroxyl ion on micro-organisms, the interaction of $\mathrm{Ca}(\mathrm{OH})_{2}$ with carbon dioxide, which alters the local environment 
for bacteria [10], and the ability of the hydroxyl ion to break down necrotic tissue [8,11,12], which may act as a substrate for bacteria. $\mathrm{Ca}(\mathrm{OH})_{2}$ has a high $\mathrm{pH}$ as a result of the release of hydroxyl ions.

Previous studies have demonstrated that $\mathrm{Ca}(\mathrm{OH})_{2}$ pastes when placed in the root canal system release the hydroxyl ion, which then diffuses through the root dentine and cementum to reach the peri-radicular tissues $[13,14]$. An important aspect of this diffusion process is to deliver the hydroxyl ion throughout the entire root canal system - that is, to all the dentine tubules, fins, transverse anastomoses, isthmuses, lateral canals, and accessory canals - since bacteria may be present in any or all of these sites. Increasing the $\mathrm{pH}$ in the dentine can therefore inhibit and destroy bacteria that remain following the mechanical procedures (instrumentation) and irrigation of the root canal during treatment. A previous study [13] showed that $\mathrm{pH}$ levels in the dentine vary according to location with inner dentine levels (i.e., adjacent to the root canal), reaching a peak of 10.8 within a few hours and then stabilizing at about $\mathrm{pH}$ 10.0. In the outer dentine, $\mathrm{pH}$ changes were much slower, taking up to three weeks to stabilize at $\mathrm{pH}$ 9.3. In that study, samples were only tested over a four-week period [13]. Hence, it is not known whether these $\mathrm{pH}$ levels are maintained in the dentine when long-term dressings are used during root canal treatment.

Commercial preparations of $\mathrm{Ca}(\mathrm{OH})_{2}$ are available as pastes using either saline (e.g., Calasept Plus—Nordiska, Ängelholm, Sweden), methyl cellulose (e.g., Pulpdent paste-Pulpdent Corporation, Watertown, MA, USA), or polyethylene glycol (PEG) (e.g., Calmix-Ozdent Pty Ltd., Castle Hill, Australia) as the base vehicle for the paste.

Ledermix paste (Haupt Pharma GmbH, Wolfratshausen, Germany) is a popular medicament in many countries [2]. It contains demeclocycline and triamcinolone as its active components. The former is a tetracycline antibiotic, which is designed to inhibit bacterial growth within the root canal system; the latter is a corticosteroid used to reduce inflammation in any remaining pulp tissue and within the periapical tissues [2]. The use of calcium hydroxide and Ledermix paste together as an inter-appointment medicament has been shown to have great promise in achieving a bacteria-free environment as well as reducing pain $[3,15-17]$ and for the control of external inflammatory root resorption in trauma cases [18]. The effects on hydroxyl ion release and diffusion when Ledermix paste is mixed with $\mathrm{Ca}(\mathrm{OH})_{2}$ pastes has not been investigated previously.

A smear layer is created when the root canal walls are instrumented with files $[19,20]$. Whilst this smear layer can be removed by using chelating agents as irrigants (such as EDTA) [19], not all clinicians remove it, even though studies have shown that its presence significantly reduces the ability of the tetracycline and corticosteroid components of Ledermix paste to diffuse through root dentine [20]. Hydroxyl ion diffusion was also inhibited in one study, although it was only tested over a seven-day period [19]. If the medicaments cannot diffuse effectively through dentine, then the anti-bacterial action of the material is likely to be reduced.

The main aim of this study was to compare hydroxyl ion release and diffusion through root dentine by measuring the $\mathrm{pH}$ when different formulations of $\mathrm{Ca}(\mathrm{OH})_{2}$ are used in combination with Ledermix paste. A second aim was to investigate whether smear layer on the root canal walls following root canal preparation affected diffusion of hydroxyl ion through dentine.

\section{Results}

\subsection{Inner Dentine $\mathrm{pH}$}

Table 1 and Figure 1 show that the inner dentine $\mathrm{pH}$ of Calmix was significantly higher than all the other medicament groups for most of the experimental period of 12 weeks (84 days). This group started by having marginally higher $\mathrm{pH}$ than the other groups and it reached a peak on Day 7 . The differences decreased from this point on, but they were still much higher at the final time point than the other groups. Of the other three groups, the $\mathrm{pH}$ of the Calmix/Ledermix group was slightly higher than the Calasept Plus/Ledermix group for Days 5-14, but subsequently they were comparable. 
The Pulpdent/smear layer group had significantly lower $\mathrm{pH}$ levels than both the Calmix/Ledermix and Calasept Plus/Ledermix groups.

Table 1. Maximum $\mathrm{pH}$ values obtained and the time taken to reach maximum $\mathrm{pH}\left(\mathrm{T}_{\max }\right)$ in the different levels of dentine.

\begin{tabular}{ccccc}
\hline Material & $\begin{array}{c}\text { Maximum pH-Inner } \\
\text { Dentine }\end{array}$ & $\begin{array}{c}\text { Maximum pH-Outer } \\
\text { Dentine }\end{array}$ & $\begin{array}{c}\mathbf{T}_{\max } \mathbf{p H}-\mathbf{I n n e r} \\
\text { Dentine }\end{array}$ & $\begin{array}{c}\mathrm{T} \text { max } \\
\text { Dentine }\end{array}$ \\
\hline Control & 7.45 & 7.46 & 2 days & 10 weeks \\
Calmix & 11.27 & 8.92 & 1 week & 8 weeks \\
Calmix/Ledermix & 9.42 & 8.98 & 4 weeks & 8 weeks \\
Calasept Plus/Ledermix & 9.39 & 8.58 & 4 weeks & 8 weeks \\
Pulpdent/smear layer & 9.96 & 8.62 & days & 8 weeks \\
\hline
\end{tabular}

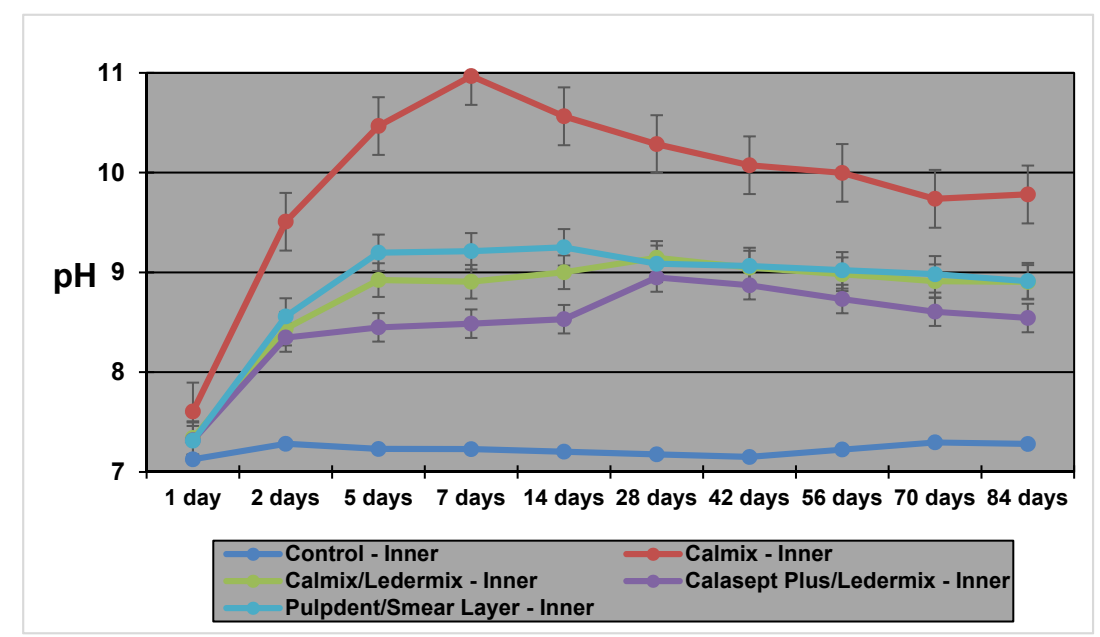

Figure 1. The $\mathrm{pH}$ values in the inner dentine with the various medicaments over 12 weeks.

\subsection{Outer Dentine $p H$}

Table 1 and Figure 2 show that the outer dentine $\mathrm{pH}$ for the Calmix group was significantly higher than all the other groups for most of the experiment. However, after 8 weeks, the difference in $\mathrm{pH}$ was much smaller when compared to the Calmix/Ledermix and Calasept Plus/Ledermix groups. The $\mathrm{pH}$ of the Calmix/Ledermix group started at a higher level than the Calasept Plus/Ledermix group and the Pulpdent/smear layer group. However, by Day 28, the difference was no longer statistically significant.

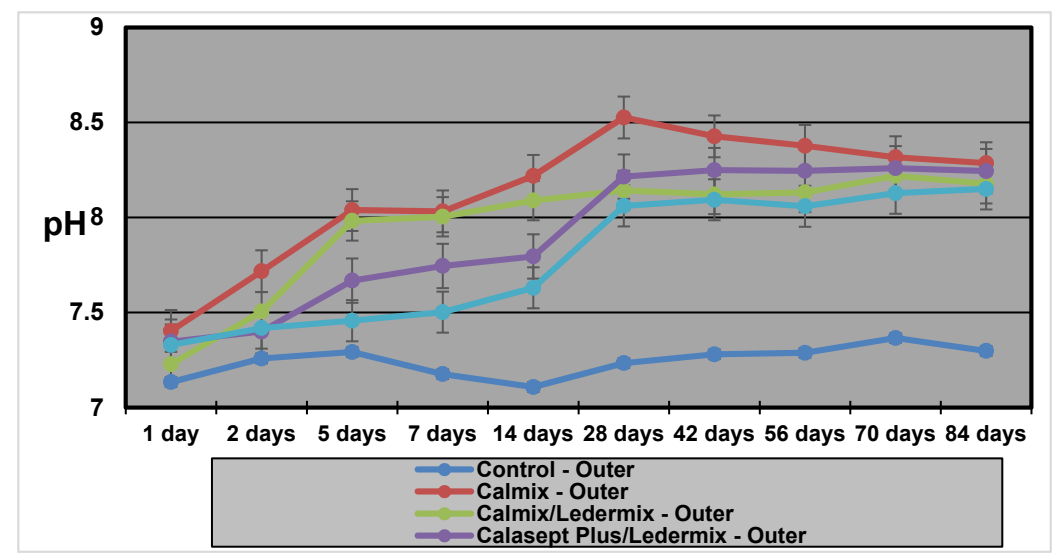

Figure 2. The $\mathrm{pH}$ values in the outer dentine with the various medicaments over 12 weeks. 


\section{3. $T_{\max }$ Comparisons}

The Calmix group exhibited a higher $\mathrm{pH}$ than the both the Calmix/Ledermix and Calasept Plus/Ledermix groups at all time intervals. The $\mathrm{T}_{\max }$ (time taken to reach maximum $\mathrm{pH}$ ) for Calmix in the inner dentine was one week compared to four weeks for both the Calmix/Ledermix and Calasept Plus/Ledermix groups. The $\mathrm{T}_{\max }$ in the outer dentine was eight weeks for all groups apart from the control group (10 weeks), but the controls had no real increase in $\mathrm{pH}$ from the baseline levels.

\subsection{Area under the Curve Analysis}

Analysis of the area under the curve across the different medicament groups (Table 2 and Figure 3) allowed the total release of hydroxyl ions to be compared. The control group, as expected, was significantly lower than all other groups in both the inner and outer dentine. Calmix had the highest amount of total release of hydroxyl ions for both the inner dentine and outer dentine measurements, and these were statistically significantly higher than the other experimental groups. The medicament group combinations that were not statistically significantly different from each other were the outer dentine measurements of the Calasept Plus/Ledermix group and the Calmix/Ledermix group, as well as the Calmix/Ledermix group compared with the Pulpdent/smear layer group.

Table 2. Area under the curve responses and statistical comparisons between groups.

\begin{tabular}{ccccccc}
\hline Medicaments & $\begin{array}{c}\text { Cavity } \\
\text { Location }\end{array}$ & Estimate & $\begin{array}{c}\text { Standard } \\
\text { Error }\end{array}$ & DF & t Value & Pr $>|\mathbf{t}|$ \\
\hline Control & Inner & 602.72 & 4.1284 & 40 & 145.99 & $<0.0001$ \\
Control & Outer & 606.24 & 4.1284 & 40 & 146.85 & $<0.0001$ \\
Calmix & Inner & 842.84 & 2.9192 & 40 & 288.72 & $<0.0001$ \\
Calmix & Outer & 694.44 & 2.9192 & 40 & 237.88 & $<0.0001$ \\
Calmix/Ledermix & Inner & 748.65 & 2.9192 & 40 & 256.45 & $<0.0001$ \\
Calmix/Ledermix & Outer & 676.98 & 2.9192 & 40 & 231.90 & $<0.0001$ \\
Calasept Plus/Ledermix & Inner & 724.92 & 2.9192 & 40 & 248.33 & $<0.0001$ \\
Calasept Plus/Ledermix & Outer & 677.28 & 2.9192 & 40 & 232.00 & $<0.0001$ \\
Pulpdent/smear layer & Inner & 754.52 & 2.9192 & 40 & 258.47 & $<0.0001$ \\
Pulpdent/smear layer & Outer & 664.51 & 2.9192 & 40 & 227.63 & $<0.0001$ \\
\hline
\end{tabular}

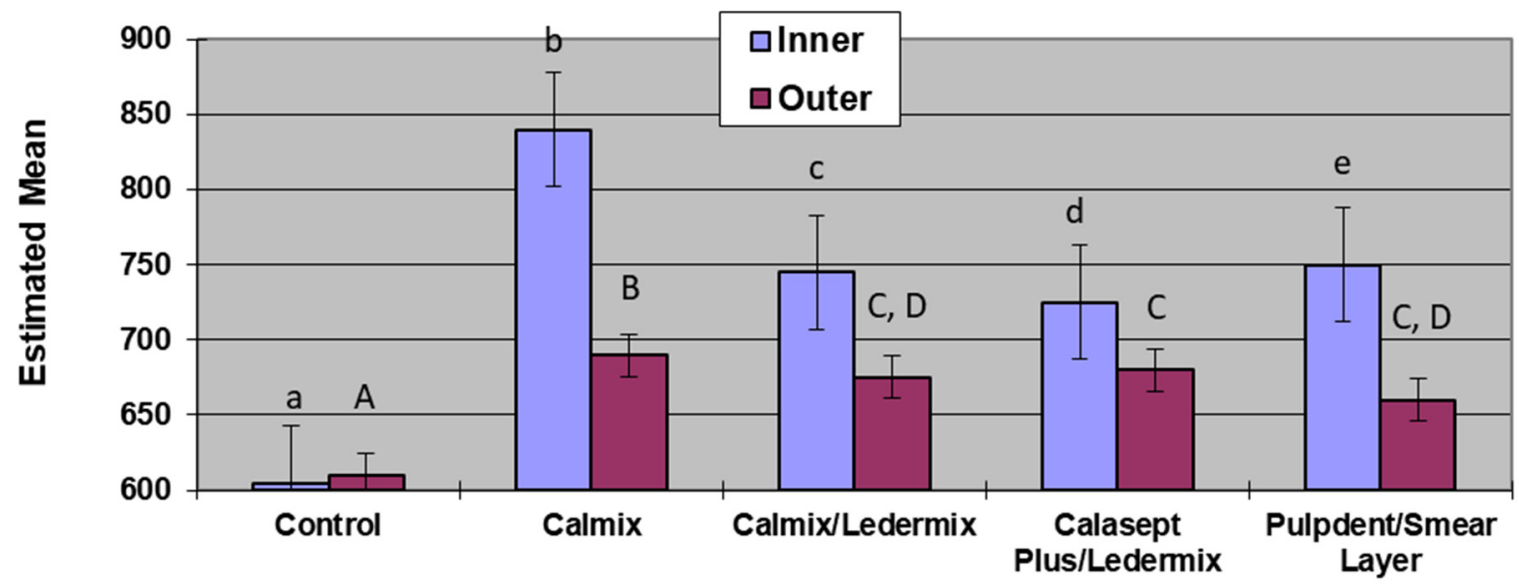

Figure 3. Area under the curve comparisons between groups. Groups with the same letter were not statistically significantly different from each other (lower case for inner dentine; upper case for outer dentine). 


\section{Discussion}

The $\mathrm{pH}$ measurements in this study demonstrate that hydroxyl ions are readily released from the various $\mathrm{Ca}(\mathrm{OH})_{2}$ formulations, even when mixed with Ledermix paste. They then diffuse through the root dentine. The results and diffusion patterns were consistent with other similar studies that have investigated $\mathrm{pH}$ changes in root dentine $[13,14]$.

Calmix, a $\mathrm{Ca}(\mathrm{OH})_{2}$ paste with a $30 \%$ PEG base had higher maximum $\mathrm{pH}$ values in both the inner (11.27) and outer dentine (8.92) than the methyl cellulose-based Pulpdent paste (10.0 and 8.6 respectively for inner and outer dentine) that was investigated in a similar model in a previous study [14]. The time taken to reach the maximum $\mathrm{pH}\left(\mathrm{T}_{\max }\right)$ in the inner dentine was faster for Calmix ( 1 week) than for Pulpdent (23 days) in the previous study. However, Calmix took 8 weeks to reach maximum $\mathrm{pH}$ in the outer dentine compared to 36 days for Pulpdent in the previous study [14]. The different paste base may explain the slower release over time for the Calmix group.

There was no difference in the maximum $\mathrm{pH}$ values or the $\mathrm{T}_{\max }$ for the two groups where Ledermix paste was mixed with two different forms of $\mathrm{Ca}(\mathrm{OH})_{2}$. However, with the variability obtained in the different groups for the $\mathrm{T}_{\max }$, it is advisable to increase the length of time between treatment appointments when $\mathrm{Ca}(\mathrm{OH})_{2}$ and Ledermix pastes are used together in order to ensure that the maximum $\mathrm{pH}$ has been obtained in the outer dentine when treating infected root canal systems.

Results for the Pulpdent/smear layer group were consistent with the results found by Foster et al. [19] although the methodology was different. The statistical analyses of both studies show that the presence of the smear layer on the root canal wall covering the dentinal tubules limited the diffusion of hydroxyl ions into the dentine tubules, and this resulted in both slower diffusion rates and lower $\mathrm{pH}$ in both the inner dentine and outer dentine. Therefore, the use of EDTAC during root canal preparation to remove the smear layer facilitates the maximum therapeutic effects of calcium hydroxide in the dentine and root canal system $[1,19]$. This is a similar finding to the effects of the smear layer on the diffusion of the active components of Ledermix paste through root dentine [20].

The model used in this experiment was an adaptation of that reported by Nerwich et al. [13] with the main modification being the measurement of $\mathrm{pH}$ at the mid-root level only rather than in the coronal and apical thirds of the roots. This modification was adopted following another similar experiment by Plataniotis and Abbott [14], who showed similar patterns of $\mathrm{pH}$ changes in mid-root cavities as that reported by Nerwich et al. [13]. Hence, the simpler experimental design was adopted.

Nerwich et al. [13] reported that the $\mathrm{pH}$ changes took up to 7 days to reach their peak in the outer dentine and then another 2-3 weeks to reach their maximum values of 9.3 and 9.0 in the cervical and apical thirds, respectively. The $\mathrm{pH}$ in the inner dentine showed more rapid changes, being evident within a few hours and peaking at 10.8 in the cervical third and 9.7 in the apical third. In that experiment, measurements were only done for 28 days and their data indicated slight rises in $\mathrm{pH}$ were still occurring at the end of the experiment. Nerwich et al. only tested one formulation of $\mathrm{Ca}(\mathrm{OH})_{2}$ paste, which had a saline base. Plataniotis and Abbott [14] tested different forms of $\mathrm{Ca}(\mathrm{OH})_{2}$-that is, a saline-based paste, a methyl cellulose-based paste, $\mathrm{Ca}(\mathrm{OH})_{2}$ powder mixed with saline, and a gutta percha point impregnated with $\mathrm{Ca}(\mathrm{OH})_{2}$. They reported similar diffusion patterns and $\mathrm{pH}$ values for the three aqueous solutions. However, the gutta percha-impregnated points did not release any significant amount of hydroxyl ion as $\mathrm{pH}$ values were not statistically different to the saline controls. Only the mid-root dentine was tested, and the measurements were taken over a 12 -week period. The current study was also limited to this time frame, which is consistent with clinical recommendations to renew intracanal dressings every three months if long-term treatment is being provided [3].

The higher $\mathrm{pH}$ values obtained when $\mathrm{Ca}(\mathrm{OH})_{2}$ was used alone may be beneficial to its bactericidal activities, although it also poses a higher potential for damaging the periodontal ligament cells [2]. Taylor et al. [17] showed that a 50:50 mixture of $\mathrm{Ca}(\mathrm{OH})_{2}$ and Ledermix pastes retained the properties that were thought to be of therapeutic benefit whilst not increasing the toxicity of the component parts to mammalian cells [17]. Although slightly lower $\mathrm{pH}$ levels were achieved in the dentine by mixing 
$\mathrm{Ca}(\mathrm{OH})_{2}$ with Ledermix paste, the difference was small, particularly in the outer dentine, so it is likely to still have bactericidal effects.

\section{Materials and Methods}

Forty-four single-rooted extracted human maxillary and mandibular canine teeth with a single root canal were used. The teeth were collected from the Dental School's collection of teeth that are used for teaching and research purposes. Such teeth are collected under the approval of the University's Human Research Ethics Committee. The root canal was assessed radiographically to determine suitability. Teeth were initially stored at room temperature in a solution of $0.9 \%$ buffered saline.

After standard endodontic access cavities in all teeth were cut, the root canals were instrumented and prepared using Hedström files to various sizes, according to the initial canal size and anatomy of the particular tooth. Irrigation was performed with a combination of $1 \% \mathrm{NaOCl}$ (EndoSure Hypochlor-Dentalife, Ringwood, Australia) and 17\% EDTAC (EDTA with 0.85\% Cetrimide) (Colgate/Orapharm, Victoria, Australia) during instrumentation. After the use of a file in the canal, the canal was irrigated with $2 \mathrm{~mL}$ of $\mathrm{NaOCl}$ or EDTAC on an alternating basis, except in Group 5 where the smear layer was intentionally created and left in place-this was achieved by only irrigating with $\mathrm{NaOCl}$ and not using EDTAC at all.

All experimental and control teeth had two cavities cut into the external root surface at the mid-root level with a size 6 flat fissure bur in a low-speed handpiece. One cavity was shallow-that is, just into the dentine on the buccal root surface (outer dentine). The other cavity was cut deep into the dentine on the lingual surface of each root (inner dentine). The outer dentine cavity was cut to an approximate depth of $0.5 \pm 0.25 \mathrm{~mm}$ from the buccal root surface. The inner cavity was cut to as deeply as possible so that it would be close to the canal walls without penetrating into the canal. The pre-operative radiographs were used as a guide to determine the depth of these cavities. The smear layer in these cavities was removed with a four minute application of $17 \%$ EDTAC. Once the teeth had been prepared in this manner, they were stored in unbuffered saline, and this solution was changed on a weekly basis throughout the experimental period.

The teeth were divided into four experimental groups $(n=10)$ and a control group $(n=4)$. The distribution of teeth into the groups was done such that an equal number of each tooth type was present in each experimental group. Each group was then randomly assigned to a medicament regime. The root canal of each tooth in the experimental groups was filled with a particular preparation of intracanal medicaments as follows: Group 1-no medicament; Group 2-Calmix; Group 3-Calmix and Ledermix paste in a 50:50 mixture; Group 4-Calasept Plus and Ledermix paste in a 50:50 mixture; and Group 5-Pulpdent with the smear layer left intact.

The medicaments were placed in the root canals using a spiral filler. The order of placement in the 50:50 mix groups was Ledermix paste first and then the $\mathrm{Ca}(\mathrm{OH})_{2}$ paste; thus, the two pastes were mixed within the canal with the spiral filler. The access cavities were filled with Cavit (ESPE Dental-Medizin GmbH \& Co., Seefeld, Germany), ensuring that the depth of the Cavit was at least $3 \mathrm{~mm}$. The teeth were then rinsed in distilled water, returned to their storage vials, and stored at $37^{\circ} \mathrm{C}$ in an incubator between all phases of the experiment.

The $\mathrm{pH}$ of the dentine was determined at the base of the two external cavities that had been cut into the inner and outer dentine at the following time intervals: 1, 2, 5, 7, 14, 21, and 28 days and then $5,6,8$, and 12 weeks following placement of the medicaments. At each time interval, the teeth were removed from the storage vials and rinsed in distilled water to remove any saline residue. The cavities and the root surfaces were blotted dry, and $2 \mu \mathrm{L}$ of distilled water was placed at the site being investigated-that is, at the base of the cavity. After $5 \mathrm{~min}$, the $\mathrm{pH}$ of the distilled water in each cavity was measured using a $\mathrm{pH}$ meter with a microelectrode (Microelectrodes Inc., Bedford, $\mathrm{NH}$, USA, MI-415, Batch number 77539 and 77540).

Two statistical models were used with the first model including time as a factor. Linear mixed models were used to establish whether or not there were any effects of the fixed factors on the $\mathrm{pH}$ 
level - that is, the medicament regime, the cavity location, and the time interval following placement. The second model used the area under the curve as the response- - linear mixed model approach was used to assess the effects on the response area under the curve with the fixed factors being the medicament regime, the cavity location, and the time interval following placement. The significance level for all statistical tests was set at the $95 \%$ confidence interval.

\section{Conclusions}

The 50:50 mix of different formulations of $\mathrm{Ca}(\mathrm{OH})_{2}$ with Ledermix paste exhibited similar release and diffusion patterns of hydroxyl ions as demonstrated by the $\mathrm{pH}$ measurements in the inner and outer dentine. The $\mathrm{pH}$ and total hydroxyl ion diffusion from these mixtures was lower than when calcium hydroxide was used alone. The diffusion patterns for Calmix alone were similar to those of other $\mathrm{Ca}(\mathrm{OH})_{2}$ pastes reported in earlier studies, but the $\mathrm{pH}$ levels reached in the inner and outer dentine were slightly higher. The smear layer inhibited the diffusion of hydroxyl ions, so this layer should be removed during root canal treatment in order to allow maximum medicament diffusion through the dentinal tubules for more predictable therapeutic effects.

Acknowledgments: The authors gratefully acknowledge the Australian Dental Research Foundation Inc., which supported this project with the Undergraduate Dental Student Vacation Research Scholarship program. The authors thank Basil Athanassiadis for supplying the Calmix medicament.

Author Contributions: Paul Abbott conceived and designed the experiments; Michael Cai performed the experiments; Paul Abbott, Michael Cai, and Jacqueline Castro Salgado analyzed the data; Paul Abbott, Michael Cai, and Jacqueline Castro Salgado wrote the paper.

Conflicts of Interest: The authors declare no direct conflicts of interest. Paul Abbott declares that he has acted on occasions as a Consultant to OzDent Pty Ltd., the manufacturers of Calmix, and the Australian suppliers of Ledermix paste. This company had no role in the design of the study, in the collection, analyses, or interpretation of data, in the writing of the manuscript, or in the decision to publish the results.

\section{References}

1. Byström, A.; Sundqvist, G. The antibacterial action of sodium hypochlorite and EDTA in 60 cases of endodontic therapy. Int. Endod. J. 1985, 18, 35-40. [CrossRef] [PubMed]

2. Abbott, P.V. Medicaments: Aids to success in endodontics. Part 1. A review of the literature. Aust. Dent. J. 1990, 35, 438-448. [CrossRef] [PubMed]

3. Abbott, P.V. Medicaments: Aids to success in Endodontics. Part 2. Clinical recommendations. Aust. Dent. J. 1990, 35, 491-496. [CrossRef] [PubMed]

4. Fava, L.R.; Saunders, W.P. Calcium hydroxide pastes: Classification and clinical indications. Aust. Dent. J. 1999, 32, 257-282. [CrossRef]

5. Byström, A.; Claesson, R.; Sundqvist, G. The antibacterial effect of camphorated paramonochlorophenol, camphoralated phenol and calcium hydroxide in the treatment of infected root canals. Endod. Dent. Traumatol. 1985, 1, 170-175. [CrossRef] [PubMed]

6. Heithersay, G. Stimulation of root formation in incompletely developed teeth. Oral Surg. Oral Med. Oral Pathol. 1970, 29, 620-630. [CrossRef]

7. Cvek, M. A clinical report on partial pulpotomy and capping with calcium hydroxide in permanent incisors with complicated crown fracture. J. Endod. 1978, 4, 232-237. [CrossRef]

8. Hasselgren, G.; Olsson, B.; Cvek, M. Effects of calcium hydroxide and sodium hypochlorite on the dissolution of necrotic porcine muscle tissue. J. Endod. 1988, 14, 125-127. [CrossRef]

9. Pashley, D.; Kakathoor, S.; Burnham, D. The effects of calcium hydroxide on dentine permeability. J. Dent. Res. 1986, 65, 417-420. [CrossRef] [PubMed]

10. Kontakiotis, E.; Nakou, M.; Georgopoulou, M. In vitro study of the indirect action of calcium hydroxide on the anaerobic flora of the root canal. Int. Endod. J. 1995, 28, 285-289. [CrossRef] [PubMed]

11. Metzler, R.; Montgomery, S. The effectiveness of ultrasonics and calcium hydroxide for the debridement of human mandibular molars. J. Endod. 1989, 15, 373-378. [CrossRef]

12. Andersen, M.; Lund, A.; Andreasen, J.O.; Andreasen, F.M. In vitro solubility of human pulp tissue in calcium hydroxide and sodium hypochlorite. Endod. Dent. Traumatol. 1992, 8, 104-108. [CrossRef] [PubMed] 
13. Nerwich, A.; Figdor, D.; Messer, H.H. pH changes in root dentin over a 4-week period following root canal dressing with calcium hydroxide. J. Endod. 1993, 19, 302-306. [CrossRef]

14. Plataniotis, E.; Abbott, P. A comparison of hydroxyl ion diffusion through root dentine from various calcium hydroxide preparations. Aust. Endod. J. 1999, 25, 151-152. [CrossRef] [PubMed]

15. Abbott, P.V.; Heithersay, G.S.; Hume, W.R. Release and diffusion through human tooth roots in vitro of corticosteroid and tetracycline trace molecules from Ledermix paste. Endod. Dent. Traumatol. 1988, 4, 55-62. [CrossRef] [PubMed]

16. Abbott, P.V.; Hume, W.R.; Heithersay, G.S. Effects of combining Ledermix and calcium hydroxide pastes on the diffusion of corticosteroid and tetracycline through human tooth roots in vitro. Endod. Dent. Traumatol. 1989, 5, 188-192. [CrossRef] [PubMed]

17. Taylor, M.A.; Hume, W.R.; Heithersay, G.S. Some effects of Ledermix paste and Pulpdent paste on mouse fibroblasts and on bacteria in vitro. Endod. Dent. Traumatol. 1989, 5, 266-273. [CrossRef] [PubMed]

18. Abbott, P.V. Prevention and management of external inflammatory resorption following trauma to teeth. Aust. Dent. J. 2016, 61 (Suppl. S1), 82-94. [CrossRef] [PubMed]

19. Foster, K.H.; Kulild, J.C.; Weller, R.N. Effect of smear layer removal on the diffusion of calcium hydroxide through radicular dentin. J. Endod. 1993, 19, 136-140. [CrossRef]

20. Abbott, P.V.; Hume, W.R.; Heithersay, G.S. Barriers to diffusion of Ledermix paste in radicular dentine. Endod. Dent. Traumatol. 1989, 5, 98-104. [CrossRef] [PubMed]

(C) 2018 by the authors. Licensee MDPI, Basel, Switzerland. This article is an open access article distributed under the terms and conditions of the Creative Commons Attribution (CC BY) license (http://creativecommons.org/licenses/by/4.0/). 